\title{
The Ettingshausen coefficient in quantum wells under the influence of laser radiation in the case of electron-optical phonon interaction
}

\author{
Dao Thu Hang, ${ }^{*}$ Dao Thu Ha, Duong Thi Thu Thanh and Nguyen Quang Bau \\ Faculty of Physics, Hanoi University of Sciences, Vietnam National \\ University, No. 334, Nguyen Trai Str., Thanh Xuan Dist., Hanoi, Vietnam
}

Received August 27, 2016; accepted September 30, 2016; published September 30, 2016

\begin{abstract}
By using a quantum kinetic equation for electrons interacting with an optical phonon, an analytic expression is obtained for the Ettingshausen coefficient (EC) in quantum wells with parabolic potential (QWPP) under the influence of laser radiation. The dependence of EC on frequency, amplitude of laser radiation, quantum wells parameters and temperature gradient is studied.

The theoretical results are numerically calculated, plotted and discussed for $\mathrm{GaAs} / \mathrm{GaAsAl}$ quantum well. Especially, when we study the dependence of EC on temperature, we realize that the EC in QWPP is $10^{2}$ times bigger than that in bulk semiconductors.
\end{abstract}

We are now more and more interested in studying the behavior of low-dimensional semiconductor systems. The motion of particles is strictly limited along the coordinates with a very narrow area of less than a few hundred $A^{0}$ and if the width is comparable with the wavelength Debroglie length of the particle, the energy spectrum and the wave function of the material will change. It leads to the appearance of size effects and alters most of the physical properties of materials [1]. As a result, the properties of low-dimensional systems such as: the Hall effect [2-4] absorption of electromagnetic waves, relative magnetoresistance, etc [5-9] are very different from the bulk semiconductors that the previous work studied [10-13]. The Ettingshausen effect which has just been researched in bulk semiconductors [14-17] is one of the electrical, magnetic and optical effects of semiconductor systems. It is a thermoelectric (or thermomagnetic) phenomenon that affects the electric current in a conductor when a magnetic field appears. Moreover, it has not been resolved in low-dimensional systems in general and in QWPP in particular. So, in this work, we use the quantum kinetic equation method that ensures high accuracy and high efficiency to get the EC in QWPP under the influence of laser radiation. We see some differences between the results obtained in this case and in the case of bulk semiconductors. Then, we estimate numerical values for the specific GaAs/GaAsAl quantum well to clarify our results.

In this research, we put our system into a parabolic potential with a constant electromagnetic field $\vec{E}_{1}=\left(0,0, E_{1}\right), \vec{B}=(0, B, 0)$ and a laser $\vec{E}(t)=\overrightarrow{E_{0}} \sin \Omega t$

*E-mail: daohangkhtn@gmail.com (where $\vec{E}_{0}$ and $\Omega$ are the amplitude and frequency of laser radiation). We establish the Hamiltonian of the electron optical phonon by using the wave function and energy spectrum of electron in a quantum well in the presence of laser radiation. Starting from the Hamiltonian and using the commutative relations of creation and annihilation operators, we obtain a quantum kinetic equation for electrons in QWPP. As a result, we have obtained the current density $\vec{J}$ and the thermal flux density $\vec{q}_{e}$ :

$$
\vec{J}=\int_{0}^{\infty} \vec{X}(\varepsilon) d \varepsilon, \quad \overrightarrow{q_{e}}=\frac{1}{e} \int_{0}^{\infty}\left(\varepsilon-\varepsilon_{F}\right) \vec{X}(\varepsilon) d \varepsilon,
$$

where:

$\vec{X}(\varepsilon)=\frac{e}{m^{*}} \sum_{N, \vec{k}_{x}} \overrightarrow{k_{x}} f_{N, \overrightarrow{k_{x}}} \delta\left(\varepsilon-\varepsilon_{N}\left(k_{x}\right)\right)$ (with $f_{N, \vec{k}_{x}}$ being an unknown electron distribution function perturbed due to the external fields and $\vec{k}_{x}=\left(k_{x}, 0,0\right)$ being the wave vector of an electron.

Through some analytical calculation, we could achieve conductivity tensors to determine the EC:

$$
P=\frac{1}{B} \frac{\sigma_{x x} \gamma_{x y}-\sigma_{x y} \gamma_{x x}}{\sigma_{x x}\left[\beta_{x x} \gamma_{x x}-\sigma_{x x}\left(\xi_{x x}-K_{L}\right)\right]}=\frac{A}{B},
$$

here $\sigma_{i k}, \beta_{i k}, \gamma_{i k}, \xi_{i k}$ are kinetic coefficients, $K_{L}$ is the lattice heat conductivity.

$$
\begin{aligned}
A= & \hbar \Omega\left\{-\left[s_{0}+n_{0} n_{1} \frac{\tau\left(\varepsilon_{F}\right)}{1+\omega_{c}^{2} \tau^{2}\left(\varepsilon_{F}\right)}\left[1-\omega_{c}^{2} \tau^{2}\left(\varepsilon_{F}\right)\right]\right] n_{0} n_{3}\right. \\
& \times \frac{\tau\left(\varepsilon_{F}+\hbar \omega_{0}+\hbar \Omega\right)}{1+\omega_{c}^{2} \tau^{2}\left(\varepsilon_{F}+\hbar \omega_{0}+\hbar \Omega\right)} \omega_{c}\left[\tau\left(\varepsilon_{F}+\hbar \omega_{0}+\hbar \Omega\right)+\tau\left(\varepsilon_{F}\right)\right] \\
& +\left[s_{0} \omega_{c} \tau\left(\varepsilon_{F}\right)+n_{0} n_{1} \frac{\tau\left(\varepsilon_{F}\right)}{1+\omega_{c}^{2} \tau^{2}\left(\varepsilon_{F}\right)} 2 \omega_{c} \tau\left(\varepsilon_{F}\right)\right] n_{0} n_{3} \\
& \left.\times \frac{\tau\left(\varepsilon_{F}+\hbar \omega_{0}+\hbar \Omega\right)}{1+\omega_{c}^{2} \tau^{2}\left(\varepsilon_{F}+\hbar \omega_{0}+\hbar \Omega\right)}\left[1-\omega_{c}^{2} \tau\left(\varepsilon_{F}+\hbar \omega_{0}+\hbar \Omega\right) \tau\left(\varepsilon_{F}\right)\right]\right\},
\end{aligned}
$$




$$
\begin{aligned}
& B=\left\{\left[s_{0}+\frac{n_{0} n_{1} \tau\left(\varepsilon_{F}\right)}{1+\omega_{c}^{2} \tau^{2}\left(\varepsilon_{F}\right)}\left[1-\omega_{c}^{2} \tau^{2}\left(\varepsilon_{F}\right)\right] \frac{n_{0} n_{3} \tau\left(\varepsilon_{F}+\hbar \omega_{0}+\hbar \Omega\right)}{1+\omega_{c}^{2} \tau^{2}\left(\varepsilon_{F}+\hbar \omega_{0}+\hbar \Omega\right)}\right.\right. \\
& \left.\times\left[1-\omega_{c}^{2} \tau\left(\varepsilon_{F}+\hbar \omega_{0}+\hbar \Omega\right) \tau\left(\varepsilon_{F}\right)\right]\right]\left[-\frac{\hbar^{2} \Omega^{2}}{T m} \frac{\tau\left(\varepsilon_{F}\right)}{1+\omega_{c}^{2} \tau^{2}\left(\varepsilon_{F}\right)}\left[n_{0} n_{3} \times\right.\right. \\
& \left.\times \frac{\tau\left(\varepsilon_{F}+\hbar \omega_{0}+\hbar \Omega\right)}{1+\omega_{c}^{2} \tau^{2}\left(\varepsilon_{F}+\hbar \omega_{0}+\hbar \Omega\right)}\left[1-\omega_{c}^{2} \tau\left(\varepsilon_{F}+\hbar \omega_{0}+\hbar \Omega\right) \tau\left(\varepsilon_{F}\right)\right]\right]^{2} \\
& +e\left[s_{0}+\frac{n_{0} n_{1} \tau\left(\varepsilon_{F}\right)}{1+\omega_{c}^{2} \tau^{2}\left(\varepsilon_{F}\right)}\left[1-\omega_{c}^{2} \tau^{2}\left(\varepsilon_{F}\right)\right]+\frac{n_{0} n_{3} \tau\left(\varepsilon_{F}+\hbar \omega_{0}+\hbar \Omega\right)}{1+\omega_{c}^{2} \tau^{2}\left(\varepsilon_{F}+\hbar \omega_{0}+\hbar \Omega\right)}\right. \\
& \left.\times\left[1-\omega_{c}^{2} \tau\left(\varepsilon_{F}+\hbar \omega_{0}+\hbar \Omega\right) \tau\left(\varepsilon_{F}\right)\right]\right]\left[\frac{\hbar^{2} \Omega^{2}}{T m e} \frac{\tau\left(\varepsilon_{F}\right)}{1+\omega_{c}^{2} \tau^{2}\left(\varepsilon_{F}\right)} n_{0} n_{3}\right. \\
& \left.\left.\times \frac{\tau\left(\varepsilon_{F}+\hbar \omega_{0}+\hbar \Omega\right)}{1+\omega_{c}^{2} \tau^{2}\left(\varepsilon_{F}+\hbar \omega_{0}+\hbar \Omega\right)}\left[1-\omega_{c}^{2} \tau\left(\varepsilon_{F}+\hbar \omega_{0}+\hbar \Omega\right) \tau\left(\varepsilon_{F}\right)\right]+K_{L}\right]\right\}, \\
& \text { where } \quad s_{0}=\frac{e L_{x}}{\pi \hbar} \sum_{N} \sqrt{\Delta_{0}} \theta\left(\Delta_{0}\right) \\
& n_{0}=\frac{e L_{x}}{4 \pi m^{*}} \frac{e^{2} \hbar \omega_{0}}{\varepsilon_{0}}\left(\frac{1}{\chi_{\infty}}-\frac{1}{\chi_{0}}\right) \frac{1}{e^{\frac{\hbar \omega_{0}}{k_{B} T}}-1} \frac{e^{2} E_{1}^{2}}{\Omega^{4} \hbar^{4}} \sum_{N, N^{\prime}} I\left(N, N^{\prime}\right) \text {, } \\
& n_{1}=\sum_{N, N^{\prime}} 4\left\{\left(\frac{e^{2} E_{1}^{2} \omega_{c}^{2}}{\hbar^{2} \omega_{0}^{4}}+\Delta_{0}\right)\left(\sqrt{\frac{\Delta_{1}}{\Delta_{0}}}-\sqrt{\frac{\Delta_{2}}{\Delta_{0}}}-\sqrt{\frac{\Delta_{3}}{\Delta_{0}}}-\Delta_{0}\right)\right. \\
& \left.-\left(\left(\frac{e^{2} E_{1}^{2} \omega_{c}^{2}}{\hbar^{2} \omega_{0}^{4}}+\Delta_{7}\right)\left(\sqrt{\Delta_{7}}+\sqrt{\Delta_{4}}\right)+\frac{e E_{1} \omega_{c}}{\hbar \omega_{0}^{2}}\left(\Delta_{7}+3 \Delta_{4}\right)\right\}\right] \\
& \times \theta\left(\Delta_{0}\right) \theta\left(\Delta_{1}\right) \theta\left(\Delta_{2}\right) \theta\left(\Delta_{3}\right) \theta\left(\Delta_{4}\right) \theta\left(\Delta_{7}\right), \\
& n_{3}=\sum_{N, N^{\prime}} 4\left[\left(\frac{e^{2} E_{1}^{2} \omega_{c}^{2}}{\hbar^{2} \omega_{0}^{4}}+\Delta_{7}\right)\left(\sqrt{\Delta_{7}}+\sqrt{\Delta_{6}}\right)\right. \\
& \left.+\frac{e E_{1} \omega_{c}}{\hbar \omega_{0}^{2}}\left(\Delta_{7}+3 \Delta_{6}\right)\right] \times \theta\left(\Delta_{6}\right) \theta\left(\Delta_{7}\right), \\
& \Delta_{0}=\frac{2 m^{*} \omega_{p}^{2}}{\hbar^{2} \omega_{o}^{2}}\left(\varepsilon_{F}-\hbar \omega_{p}\left(N+\frac{1}{2}\right)\right) \text {, } \\
& \Delta_{1}=\frac{2 m^{*} \omega_{p}^{2}}{\hbar^{2} \omega_{o}^{2}}\left(\varepsilon_{F}-\hbar \omega_{0}-\hbar \omega_{p}\left(N^{\prime}+\frac{1}{2}\right)\right) \text {, } \\
& \Delta_{2}=\frac{2 m^{*} \omega_{p}^{2}}{\hbar^{2} \omega_{o}^{2}}\left(\varepsilon_{F}-\hbar \omega_{0}+\hbar \Omega-\hbar \omega_{p}\left(N^{\prime}+\frac{1}{2}\right)\right), \\
& \Delta_{3}=\frac{2 m^{*} \omega_{p}^{2}}{\hbar^{2} \omega_{o}^{2}}\left(\varepsilon_{F}-\hbar \omega_{0}-\hbar \Omega-\hbar \omega_{p}\left(N^{\prime}+\frac{1}{2}\right)\right), \\
& \Delta_{4}=\frac{2 m^{*} \omega_{p}^{2}}{\hbar^{2} \omega_{o}^{2}}\left(\varepsilon_{F}-\hbar \omega_{0}-\hbar \omega_{p}\left(N+\frac{1}{2}\right)\right) \text {, } \\
& \Delta_{5}=\frac{2 m^{*} \omega_{p}^{2}}{\hbar^{2} \omega_{o}^{2}}\left(\varepsilon_{F}-\hbar \omega_{0}-\hbar \Omega-\hbar \omega_{p}\left(N+\frac{1}{2}\right)\right) \text {, } \\
& \Delta_{6}=\frac{2 m^{*} \omega_{p}^{2}}{\hbar^{2} \omega_{o}^{2}}\left(\varepsilon_{F}-\hbar \omega_{0}+\hbar \Omega-\hbar \omega_{p}\left(N+\frac{1}{2}\right)\right) \text {, } \\
& \Delta_{7}=\frac{2 m^{*} \omega_{p}^{2}}{\hbar^{2} \omega_{o}^{2}}\left(\varepsilon_{F}-\hbar \omega_{p}\left(N^{\prime}+\frac{1}{2}\right)\right) \text {, }
\end{aligned}
$$

$k_{B}$ is the Boltzmann constant, $L_{x}$ and $\varepsilon_{F}$ are the normalized length in the $x$ direction and the Fermi level, respectively.

The EC $(2,3,4)$ exhibits also its dependence on external fields (i.e. electrical field intensity $\vec{E}_{1}$, the cyclotron frequencies $\omega_{c}$ ), including laser radiation (i.e. frequency $\Omega$ and amplitude $\vec{E}_{0}$ of laser), temperature and special parameters for QWPP (i.e. quantum well length $L_{x}$ ). In the next section, we will give numerical calculations carried out for a specific GaAs/GaAsAl Quantum Well to discuss the dependence more clearly.

In this section, we present detailed numerical calculations of the EC in QWPP subjected to uniform crossed magnetic and electric fields under the influence of laser radiation .The parameters used in the calculations are as follows:

$$
\varepsilon_{F}=50 m e V, \chi_{\infty}=10,9, \quad \chi_{0}=12,9, \quad m=0.067 \times m_{0}
$$

( $m_{0}$ is the mass of a free electron) and for the sake of simplicity: $\hbar \omega_{0}=36.25 \mathrm{meV}, \quad \tau=10^{-12}$. We only consider electron transition between the ground state and the lowest excited state, respectively: $\mathrm{N}=0, \mathrm{~N}=1$, electron transition amongst other states is not considerable.

Figure 1 describes the dependence of the EC on laser frequency with

$$
E_{1}=5.10^{2} \mathrm{Vm}^{-1}, E_{0}=5.10^{6} \mathrm{Vm}^{-1}, L_{x}=2.10^{9} \mathrm{~m}, \mathrm{~T}=100 \mathrm{~K} \text {. }
$$

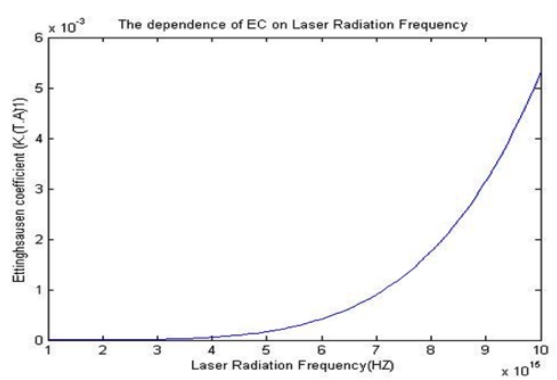

Fig. 1. The dependence of EC on laser frequency.

In Figure 1, we show the dependence of the EC on the frequency $\Omega$ of intense laser radiation.

The electrons will absorb photons and lead to a change in the energy spectrum of a material that makes the EC change. From these figures, we can see that when the frequency of laser radiation increases, the EC increases. 


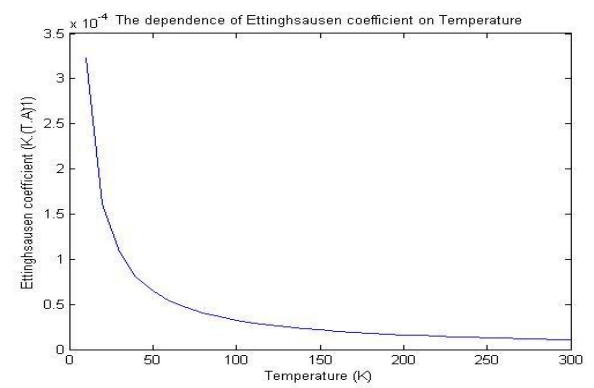

Fig. 2. The dependence of EC on temperature.

Figure 2 describes the dependence of the EC on temperature with

$$
\begin{aligned}
& E_{1}=5.10^{2} \mathrm{Vm}^{-1}, E_{0}=5.10^{6} \mathrm{Vm}^{-1}, \Omega=9.10^{16}(\mathrm{~Hz}), \\
& L_{x}=2.10^{9} \mathrm{~m} .
\end{aligned}
$$

In Figure 2, the dependence of the EC on temperature is non-linear and decreases to zero when temperature increases. The directionality of an outside electromagnetic wave is less remarkable because an electron is more chaotic with great velocity when temperature is higher. The dependence of the EC on temperature under the influence of laser radiation which we obtained in QWPP is similar to the results published before in the case of bulk semiconductors. However, the value of the EC in QWPP obtained in this case is $10^{2}$ times larger than the EC in bulk semiconductors [15].

Figure 3 describes the dependence of the EC on quantum well length with

$$
E_{1}=5.10^{2} \mathrm{Vm}^{-1}, E_{0}=5.10^{6} \mathrm{Vm}^{-1}, \mathrm{~T}=100, \Omega=9.10^{16}(\mathrm{~Hz}) .
$$

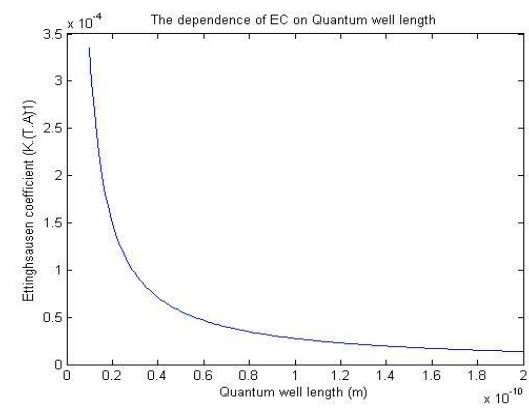

Fig. 3. The dependence of the EC on quantum well length.

Figure 3 shows that the dependence of the EC on quantum well length is non-linear and decreases to zero when quantum well length increases. This result is completely consistent with the previously studied theory. It means that when quantum well length approaches infinity, it returns to the case of bulk semiconductors. The reason is that when the quantum well length increases, our material approaches to the bulk semiconductor structure. Therefore, the EC lessens quickly.
In this paper, we analytically investigated the EC in QWPP in the presence of a magnetic field. The electronphonon interaction is taken into account at low temperatures, and for non-degenerated electron gas.

Basing on our new analytical expression of the EC in the quantum wells with parabolic potential and under the electron - optical phonon scattering mechanism, we realize that the EC depends on some elements such as: the parameters of quantum wells, amplitude and frequency of radiation, phonon frequency and temperature.

We estimated numerical values and graph for a GaAs/GaAsAl quantum wells to see clearly the nonlinear dependence of the EC on laser radiation frequency. The more laser radiation frequency increases, the more the EC increases. However, the EC reduces immediately if temperature or quantum wells length increases. We concluded the results originated from reduced dimensions effects and the influence of laser radiation on the EC. Especially, when we studied the dependence of the EC on temperature, we realized that the EC in QWPP was $10^{2}$ times bigger than that in bulk semiconductors. This was the difference between the EC in QWPP and the EC in bulk semiconductors.

This research is completed with the financial support from the Vietnam NAFOSTED (N0 103.01-2015.22).

\section{References}

[1] A. Shik, Quantum wells in Physics and Electronics of twodimensional systems (World Scientific, 1999).

[2] N.Q. Ba, B.D. Hoi, Int. J. Mod. Phys. B 281450001 (2014).

[3] N.Q. Bau, B.D. Hoi, J. Korean Phys 60, 59 (2012).

[4] T.C. Phong, N.Q. Bau, J. Korean Phys 42, 647 (2003).

[5] N.Q. Bau, L.T. Hung, N.D. Nam, J. Electromagn. Waves Appl. 24, 1751, (2010).

[6] N.Q. Bau, H.D. Trien, J. Korean Phys. Soc. 56 , 120 (2010).

[7] S.G.K. Yua, K.W. Kim, M.A. Stroscio, G.J. Iafrate, A. Ballato, J. Korean Phys 80, 2815 (1996).

[8] M. Meziani, J. Lusakowski, W. Knap, N. Dyakonova, F. Teppe, K.Romanjek, M. Ferrier, R. Clerc, G. Ghibaudo, F. Boeuf, T. Skotnicki, J. Appl. Phys. 96, 5761 (2004).

[9] N. Nishiguchi, Phys. Rev 52, 527 (1995).

[10] P. Zhao, Sov.Phys. 49, 13589 (1994).

[11] V.V.Pavlovich, E.M. Epshtein, Sov. Phys. Semicond 11, 809 (1977).

[12] A.A. Pankratov, E.M. Epshtein, Sov. Phys. Semicond. [Fiz. Tekh. Poluprovodn.] 16, 1689 (1982).

[13] R. Shmit, Semiconductors (Mir, Moskva 1982).

[14] B.V. Paranjape, J.S.Levinger, Phys. Rev 120, 437 (1960).

[15] V.L. Malevich, E.M. Epshtein. Sov. Phys. Solid State [Fiz. Tverd. Tela ] 18, 1286 (1976).

[16] V.L. Malevich, E.M.Epshtein, Sov. Phys 18, 230 (1976).

[17] E.M. Epshtein, Sov. Phys 120, 226 (1976). 